\title{
SUBPIXEL MAPPING OF HYPERSPECTRAL IMAGE BASED ON LINEAR SUBPIXEL FEATURE DETECTION AND OBJECT OPTIMIZATION
}

\author{
Zhaoxin $\mathrm{Liu}^{1}$, Liaoying Zhao ${ }^{1}$, Xiaorun $\mathrm{Li}^{2 *}$, Shuhan Chen ${ }^{2}$ \\ ${ }^{1}$ Institute of Computer Application Technology, Hangzhou Dianzi University, China - ( 162050131, zhaoly)@ hdu.edu.cn \\ ${ }^{2}$ College of Electrical Engineering, Zhejiang University, China - (lxr, 11410057)@ zju.edu.cn
}

KEY WORDS: Hyperspectral Imagery, Linear Subpixel Feature Detection, Subpixel Mapping, Space Correlation,Template Matching

\begin{abstract}
:
Owing to the limitation of spatial resolution of the imaging sensor and the variability of ground surfaces, mixed pixels are widesperead in hyperspectral imagery. The traditional subpixel mapping algorithms treat all mixed pixels as boundary-mixed pixels while ignoring the existence of linear subpixels. To solve this question, this paper proposed a new subpixel mapping method based on linear subpixel feature detection and object optimization. Firstly, the fraction value of each class is obtained by spectral unmixing. Secondly, the linear subpixel features are pre-determined based on the hyperspectral characteristics and the linear subpixel feature; the remaining mixed pixels are detected based on maximum linearization index analysis. The classes of linear subpixels are determined by using template matching method. Finally, the whole subpixel mapping results are iteratively optimized by binary particle swarm optimization algorithm. The performance of the proposed subpixel mapping method is evaluated via experiments based on simulated and real hyperspectral data sets. The experimental results demonstrate that the proposed method can improve the accuracy of subpixel mapping.
\end{abstract}

\section{INTRODUCTION}

Hyperspectral images have become one of the most important information sources for earth observation. However, mixed pixels are widespread in the images due to the fact that hyperspectral images generally have low spatial resolution. Spectral unmixing provides the abundances of pure spectral signals for each pixel, without providing spatial distribution at subpixel level (Chang, 2017).It is difficult for traditional classification technologies to accurately determine the spatial distribution of ground features because more than one ground is contained in a mixed pixel (Peijun et al., 2016). Subpixel mapping (SMP) technology is an effective method to solve this problem.

A variety of SMP algorithms, such as linear optimization techniques (Chen et al., 2017, A.Erturk., 2014), Markov random field (Liu and Trinder, 2016), pixel-swapping (Atkinson, 2005), subpixel/pixel spatial attraction model (Mertens et al., 2006), Hopfield neural networks (Wang et al., 2016), BP neural networks (Shi et al., 2014) and geo-statistics have been proposed and developed over the last decade (Boucher and Kyriakidis, 2006). Among these, the linear optimization technique, which is based on pixels spatial distribution relevance theory, has grown popular on account of its simplicity and efficient performance. To date, most linear optimization SPM methods can perform well for predicting the spatial distribution in high resolution (H-resolution), where the objects of interest are larger than the pixels. Furthermore, the linear subpixel phenomenon is also very important for remote sensing image interpretation (Fisher, 1997, Xu et al., 2014). For example, it is important for land cover classes with linear subpixel features, such as roads and rivers. Applying linear optimization methods to fine linear objects where the pixels are both shorter and wider than the objects of interest, may lead to loss of linear connectivity for linear objects; even for larger linear objects, it may lead to unsmooth boundaries (Ge et al., 2016). To address these problems, an improved pixel-swapping algorithm (PSA), namely, linearized PSA, has been proposed to map rural linear

\footnotetext{
${ }^{*}$ Corresponding author
}

land cover features (Thornton et al., 2007). However, the type of mixed pixel cannot be automatically determined and is limited to land cover classes that only contain the linear subpixel type. $\mathrm{Xu}$ et al. (Xu et al., 2014) proposed an adaptive SMP framework based on a multi-agent system for remote sensing images. However, this method cannot be guaranteed to obtain the best result because it is difficult to determine the rotation angle. Ge et al. (Ge et al., 2016) classify geographical objects into areal, linear, and point patterns according to their spatially geometric characteristics and using different methods to deal with SMP for different object patterns. However, this method fails to effectively detect the linear subpixel feature.

To address these problems, this paper proposes a new SMP algorithm based on linear subpixel feature analysis and linear optimization. The linear subpixel features are detected based on maximum linearization index analysis, and the linear subpixel is mapped by template matching method. The final results are obtained by the method based on both perimeter minimum and binary quantum particle swarm optimization. The remainder of this paper is organized as follows. Section 2 introduces the proposed SPM strategy. Section 3 contains the experimental results and analysis. Section 4 concludes this paper.

\section{PROPOSED METHOD}

The entire procedure contains three main steps: spectral unmixing, feature detection, and subpixel mapping. Since spectral unmixing is not the emphasis of this paper, the rest of this section will highlight the theorem of linear subpixel feature detection and the procedure of subpixel mapping. The flowchart of the proposed method is shown in Figure 1.

\subsection{Linear Subpixel Feature Detection}

In order to determine if there is a linear subpixel feature in a mixed pixel, it is necessary to consider its neighboring pixels. Assuming that the target mixed pixel is denoted as $P_{i j}$, the $D \times D$ neighborhood pixels for $P_{i j}$ forms a set of pixels 


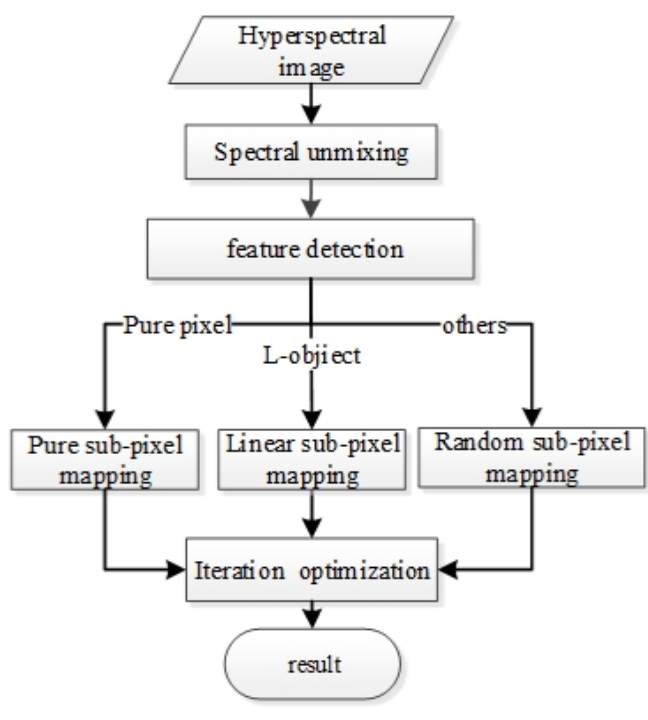

Figure 1. The flowchart of the proposed method

$$
E_{i j}=\left\{P_{i^{\prime} j^{\prime}}|| i^{\prime}-i\left|\leq \frac{D}{2} \cap\right| j^{\prime}-j \mid \leq \frac{D}{2}\right\}
$$

where $\cap$ means the "and" operator. Let each pixel represent a $1 \times 1$ grid square, then all pixels in $E_{i j}$ form a square region of length $D$. Defining a new uniform coordinate system and setting the origin at the upper left, then every pixel $p_{i j}$ will receive a coordinate $(i+0.5, j+0.5)$. In this new coordinate system, the region of the grid square for pixel $P_{i^{\prime} j^{\prime}}$ is $\left(i^{\prime} \rightarrow\left(i^{\prime}+1\right)\right) \cap$ $\left(j^{\prime} \rightarrow\left(j^{\prime}+1\right)\right)$, which is represented as $E R_{i^{\prime} j^{\prime}}$, and the set of all $A R_{i^{\prime} j^{\prime}}$ for pixels in $E_{i j}$ is

$$
C A_{i j}=\left\{A R_{i^{\prime} j^{\prime}} \mid\left(i^{\prime} \rightarrow(i+1)\right) \cap\left(j^{\prime} \rightarrow(j+1)\right),\left(i^{\prime}, j^{\prime}\right) \in E_{i j}\right\}
$$

where $C A_{i j}$ is referred to the control area of pixel $p_{i j}$.

To determine whether a linear subpixel feature exists in the mixed pixel, every possible straight line across the center of the pixel should be verified. Taking $D=5$ as an example, Fig. 2 describes how to determine whether a linear subpixel feature of class $c$ exists in the mixed pixel $p_{i j}$, where the pixels contained class $\mathrm{C}$ are marked with a yellow grid and $\vartheta$ is the angle between the line $L_{\lambda 7}$ and $L_{b}$. The decision procedure involves three steps.

Firstly, find all possible lines. Any line in the picture can be represented by pixels it passes through, and the task is to find all the pixel sets. As Fig. 2 shows, lines between $L_{a}$ and $L_{\lambda 7}$ occupy the same pixels, therefore lines between $L_{\lambda 7}$ and $L_{-\lambda 7}$ can delegate all possible lines. All solid lines in Fig. 2 are drawn by connecting the center of pixel $p_{i j}$ with each grid vertex of the control area $C A_{i j}$, and the angle between the $\frac{(D+1)^{2}}{4}$ lines and $L_{b}$ is

$\beta=\arctan \left(\frac{m+0.5}{n+0.5}\right), m=0,1, \cdots, \frac{D-1}{2}, n=0,1, \cdots, \frac{D-1}{2}$

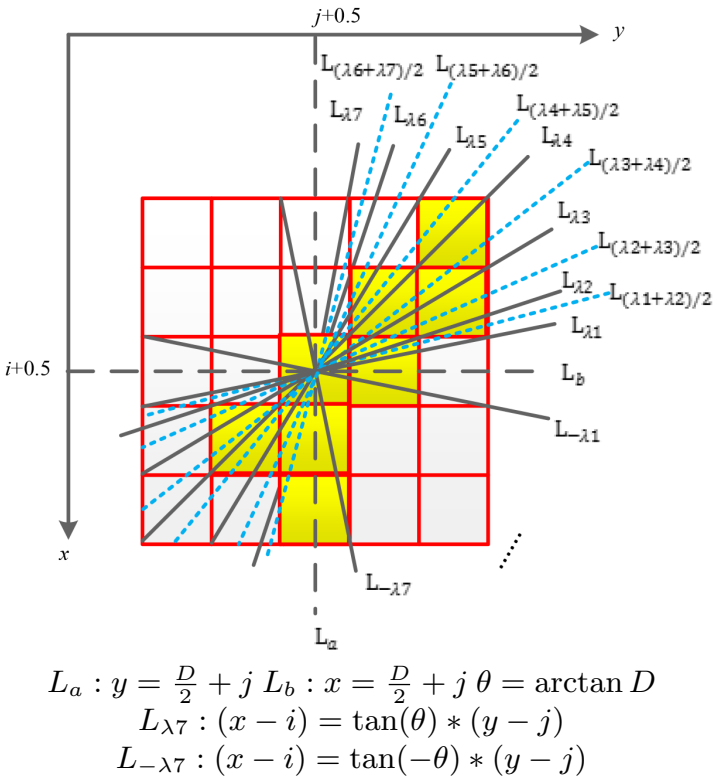

Figure 2. Sketch map of the linear subpixel feature detection for class $c$ in $p_{i j}$

where $\beta_{0,0}=\beta_{1,1}=\cdots=\beta_{\frac{D}{2}, \frac{D}{2}}$. when the duplicated lines are removed, the total number of lines is $p=\frac{(D+1)^{2}}{4}-\frac{(D+1)}{2}+1$. Sort the angles of the $p$ lines in ascending order to be reformulated as $\lambda_{1}<\lambda_{2}<\cdots<\lambda_{p}$. Obviously, the pixel sets corresponding to the $p$ lines are all varied. Furthermore, any lines between $L_{\lambda_{i}}$ and $L_{\lambda_{i+1}}$ occupy the same pixels as the line with angle $\frac{\lambda_{i}+\lambda_{i+1}}{2}$. Therefore, the entire set for the angle of the lines passing through $P_{i j}$ in $C A_{i j}$ is

$\beta=\left\{ \pm \lambda_{i} \mid i=1, \cdots p\right\} \cup\left\{ \pm \frac{\lambda_{j}+\lambda_{j+1}}{2} \mid j=1, \cdots p-1\right\}$

where the $\cup$ symbol represents the "or" operator.

Secondly, for every line $L_{\lambda}$, find the intersection of the corresponding pixel set and $C A_{i j}$.

$$
P L_{\lambda}=\left\{P_{i^{\prime} j^{\prime}} \mid A R_{i^{\prime} j^{\prime}} \cap L_{\lambda} \neq O, A R_{i^{\prime} j^{\prime}} \in C A_{i j}\right\}
$$

Finally, use the linear subpixel feature detection rule in (Xu et al., 2014) to determine if there is a linear subpixel feature in $P_{i j}$. The rule is sketched in the following as

If $M L I \geq \rho$ and $G_{k^{\prime}}\left(c^{\prime}\right) \geq D$ was satisfied, then there is a linear subpixel feature in the pixel $P_{i j}$, where $\rho$ is the threshold to determine if there is a linear subpixel feature, and

$G_{\lambda}(c)=\sum_{p_{i^{\prime} j^{\prime}} \in P L_{\lambda}} g_{i^{\prime} j^{\prime}}, g_{i^{\prime} j^{\prime}}=\left\{\begin{array}{cc}1 & S_{c}\left(i^{\prime}, j^{\prime}\right) \\ 0 & \text { else }\end{array} \quad, P_{i^{\prime} j^{\prime}} \in P L_{k}\right.$ 
$G_{k}(c)$ is the number of pixels in $P L_{\lambda}$ for class $c$ whose fraction values are greater than zero, $M L I$ (maximum linearization index) is defined as

$$
M L I=\varnothing_{k^{\prime}}\left(c^{\prime}\right), \exists k^{\prime}, c^{\prime}: \varnothing_{k^{\prime}}\left(c^{\prime}\right)=\max \left\{\varnothing_{k}(c), c \in C\right\}
$$

$$
\phi_{k}(c)=\frac{G_{k(c)}}{H(c)}
$$

$\phi_{k}(c)$ denotes the possibility of a line existing for class $c$, and

$$
H(c)=\sum_{P} \sum_{\substack{i^{\prime} j^{\prime} \\
i^{\prime}}} h_{i^{\prime}, j^{\prime}}, h_{i^{\prime}, j^{\prime}}=\left\{\begin{array}{cc}
1, & S_{c}\left(i^{\prime}, j^{\prime}\right)>0 \\
0, & \text { else }
\end{array} P_{i^{\prime}, j^{\prime}} \in E_{i j}\right.
$$

where $S_{c}\left(i^{\prime}, j^{\prime}\right)$ is the fraction value of class $c$ in pixel $P_{i^{\prime} j^{\prime}}$.

Referring to (Xu et al., 2014), in this paper, the parameter $\rho$ for the linear subpixel feature detection was set to the experiential value of 0.5 .

\subsection{Subpixel Mapping}

Apply the template matching method (Ge et al., 2016) to realize the linear subpixel mapping. The templates are represented by a binary matrix. Taking scale factor 3 as example, the binary matrix is

$$
T_{k}=\left[\begin{array}{ccc}
t(-1,1) & t(-1,0) & t(-1,1) \\
t(0,-1) & t(0,0) & t(0,1) \\
t(1,-1) & t(1,0) & t(1,1)
\end{array}\right]
$$

where $T_{k}$ denotes the template $k, t(m, n) \in\{0,1\}, m, n=$ $-1,0,1$, the elements $t(m, n)=1$ represent the template, and the elements $t(m, n)=0$ represent the background.

Calculate the correlation coefficient between a template and a local subset of an image, which is defined as

$r_{i j, k}=\frac{\sum_{m=-w}^{w} \sum_{n=-w}^{w} T_{k}(m, n) \cdot S_{c}(i+m, j+n)}{\sqrt{\sum_{m=-w}^{w} \sum_{n=-w}^{w} S_{j c}(i+m, j+n)^{2} \cdot \sum_{m=-w}^{w} \sum_{n=-w}^{w} T_{k}(m, n)^{2}}}$

where $w=\frac{s-1}{2}, T_{k}(m, n)$ is the element of the template $T_{k}$. $S_{c}$ is the fraction value of class $c$. The template with the highest $r_{i j, k}$ is selected as the optimal template.

In order to assign the land cover class to each subpixel to the closest optimal template, the Euclidean distance is calculated between any subpixel and each element that has the value 1 in the optimum template after combining the coordinates of pixel$\mathrm{s}$, templates, and subpixels into a unified coordinate system. The $S_{c}(i, j) \times s^{2}$ subpixels of the shortest distance are allocated to the land cover class $c$. It should be noted that, if the mixed pixels $P_{i j}$ only have two kinds of land cover classes, then the other subpixel
$P_{a, b}$ in the $P_{i j}$ class can be determined directly. If the land cover classes number in $P_{i j}$ is greater than two, then the remaining subpixel classes will determined by pixel attraction.

After the initial subpixel mapping for pixels with different features, an iteration optimization procedure is carried out for global subpixel. The perimeter of the areas belonging to the same class is chosen as the object optimization function, which is described as

$$
C=\sum_{i=1}^{M} \sum_{j=1}^{C_{i}} p_{i j}
$$

where $M$ is the number of the classes, $C_{i}$ is the number of connected components of the class $i$, and $P_{i j}$ is the perimeter of the connected component $j$.

The modified binary quantum particle swarm optimization algorithm (MBQPSO) (Chen et al., 2017) is used to obtain the final result.

\section{EXPERIMENTS RESULTS}

Both the simulation image and real image are used to evaluate the performance of the proposed subpixel mapping method in dealing linear feature object. We compared the subpixel mapping performance of proposed method, the binary particle swarm optimization algorithm (Chen et al., 2017), and the shape density index method (Ge et al., 2016). For convenience, the three methods are abbreviated as CLS_BQPSO, BQPSO, and SDI_BQPSO, respectively. The classification accuracy assessment was undertaken using the indices of visual interpretation, Kappa coefficient (kappa), CPU time and recognition rate.

\subsection{Simulation Experiment}

The ground truth map with size of $270 \times 180$ pixels is shown in Fig.3(a). This map contains seven land cover classes labeled with different colors. Seven kinds of feature spectrals are selected from the USGS spectral library to generate a dataset based on the ground truth, with the resulting dataset downsampled by $3 \times 3$ to obtain the simulated hyperspectral image. The $30^{t h}$ band grey map of the simulated dataset is shown in Fig.3(b).

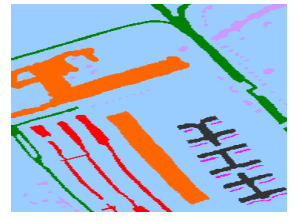

(a)

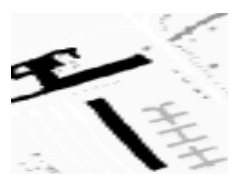

(b)
Figure 3. Simulated hyperspectral imagery :(a) True distribution, (b) $30^{\text {th }}$ band grey map.

The endmember spectral was extracted by FNSGA (Zhao et al., 2015) and the fraction maps were generated by Fully Constrained Least Squares (Heinz and Chein-I-Chang, 2002) method on the degraded low-resolution images. Then subpixel localization was performed. All of experiments were implemented using an Intel(R) Core(TM) i5-2380P CPU with the MATLAB R2014a software package. 


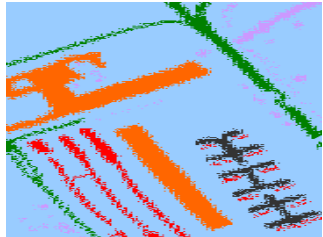

(a)

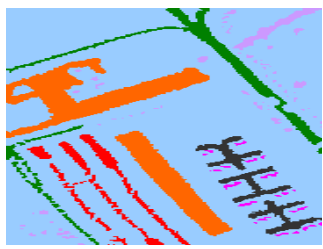

(c)

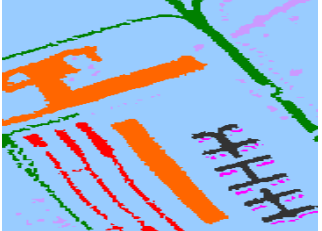

(b)

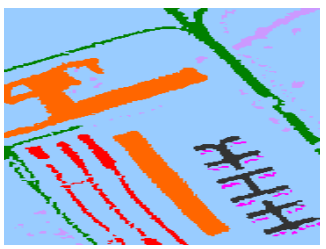

(d)
Figure 4. Subpixel mapping results of three algorithms : (a) initialization,(b) BQPSO,(c) CLS_BQPSO,(d) SDI_BQPSO.

\begin{tabular}{llcl}
\hline Algorithm & $\begin{array}{l}\text { Recognition } \\
\text { rates (\%) }\end{array}$ & Kappa coefficient & $\begin{array}{l}\text { Time } \\
(\mathrm{s})\end{array}$ \\
\hline BQPSO & 97.06 & 0.9263 & 133 \\
CLS_BQSPO & 98.09 & 0.9516 & 140 \\
SDI_BQPSO & 97.13 & 0.9271 & 134 \\
\hline
\end{tabular}

Table 1. Accuracy assessments of the three methods

Fig.4 (b)-(d) shows that spatial locations of the entire image. As can be observed from Fig.4, many linear features exist, but the straight-line boundary is not smooth and has a number of serrations, influencing the linear feature positioning. Despite the above effects, Fig.6 (c) and (d) shows a greater connectivity in each class, which made CLS_BQPSO and SDI_BQPSO produce comparable high recognition rate and kappa in Table.1. In contrast, BQPSO lost some connectivity and resulted in unrealistically jagged boundaries in Fig.4 (b), because it treats all the features as area objects. The result of SDI_BQPSO is not as good as CLS_BQPSO because CLS_BQPSO considered all linear subpixel features.

When compared with other two methods, the CLS_BQPSO method has the best performance and it shows the highest recognition rate and kappa, as can be observed in Table.1. CLS_BQPSO outperforms BQPSO and SDI_BQPSO, not only for the linear subpixel features, but also for when the neighborhood pixel attraction is considered in the linear feature mixed pixel mapping. The running time is longer on account of the fact that the latter two algorithms need to separately determine whether the mixed pixel has a linear subpixel feature.

The simulated experiment confirms the competitive performance of the proposed algorithm from both qualitative and quantitative aspects.

\subsection{Real Image Experiment}

The experimental data used ROSIS hyperspectral remote sensing images collected at the Italian University of Pavia. The size of image is $610 \times 340$ pixels, including nine classes, the number of bands is 103 and the geometric resolution is $1.3 \mathrm{~m}$. Intercept
$117 \times 75$ size of the this area, the region contains five classes, where Fig.5(a) was the true distribution, Fig.5(b) was the grey map of band 60 after smoothing. This area contains five land cover classes labeled with different colors. The abundance is adjusted, and the pixel whose minimum value of the difference between the maximum abundance and other abundance is greater than a certain threshold is used as the pure pixel. Because the metal region is obvious, only the position of the metal region is counted recognition rate, the experiment found that the threshold was 0.4 .

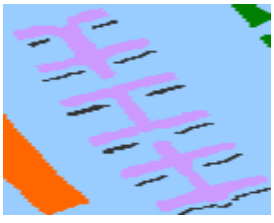

(a)

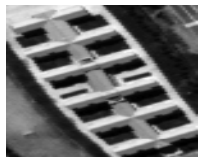

(b)
Figure 5. True hyperspectral dates : (a)True distribution, (b) $60^{t h}$ band grey image.

Fig.6 presents the different subpixel mapping results. As we can see, the subpixel mapping results of CLS_BQPSO, BQPSO, and SDI_BQPSO appear similar, and the maps have many isolated points. Table. 2 tabulates the results of different methods. By examining results in Table.2, we can also find the result of CLS_BQPSO is better than the other methods in terms of recognition rate and kappa. The BQPSO method, together with the SDI_BQPSO algorithm, obtains relatively low values.

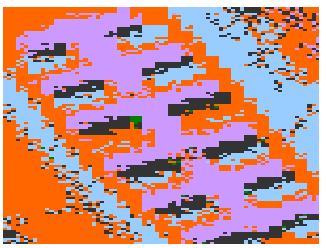

(a)

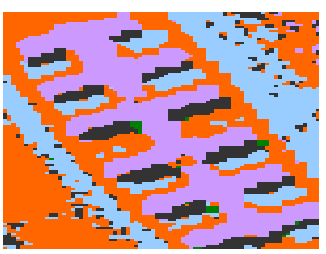

(c)

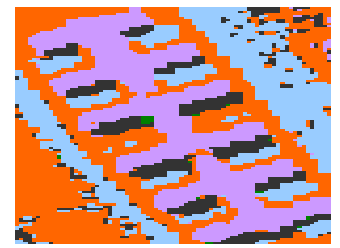

(b)

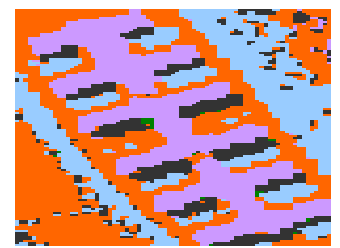

(d)
Figure 6. Subpixel mapping results of three algorithms : (a) initialization,(b) BQPSO,(c) CLS_BQPSO,(d) SDI_BQPSO.

\begin{tabular}{lcl}
\hline Algorithm & Recognition rates (\%) & Time (s) \\
\hline BQPSO & 97.84 & 40.37 \\
CLS_BQSPO & 98.52 & 42.19 \\
SDI_BQPSO & 98.01 & 40.63 \\
\hline
\end{tabular}

Table 2. Accuracy assessments of the three methods

From Table 2, it can be observed that the overall recognition rate of the algorithm for linear mixed pixel judgment and location is still slightly higher than that of BQPSO without linear judgment mapping. This is owing to the fact that the traditional subpixel mapping method used with linear features will make the linear 
features lose their connectivity, resulting in reduced spatial dependence. Moreover, the running time of CLS_BQPSO is the longest because it needs to determine whether the mixed pixel contains subpixel-level linear features, while the running time of SDI_BQPSO is slightly lower compared to BQPSO because it determines the line features based on shape density index. The running time of BQPSO is the shortest as a consequence of it not needing the above judgments and mapping.

\section{CONCLUSION}

In this paper, we have proposed a new method for subpixel mapping. Considering the deficiency that the traditional subpixel mapping only considers maximizing the spatial dependence, while ignoring the other structures in mixed pixels, such as linear subpixel features, this paper combines the judgment of linear subpixel feature and matching localization, and makes corresponding changes. In doing so, highly accurate results for remote sensing was achieved. We propose a new straight-line judgment mechanism to avoid the inappropriate choice of the rotation angle in linear subpixel feature detection affecting the linear subpixel feature judgment result. In particular, neighborhood pixel attraction is considered when mapping linearly mixed pixels. In this way, not only are the subpixel localization of the linear subpixel features considered, but the subpixel mapping of the area features are also considered, thereby improving the subpixel mapping accuracy as a whole.

\section{ACKNOWLEDGEMENTS}

This work was supported by The National Natural Science Foundation of China (no.61671408,no.61571170) and The Joint Funds of the Ministry of Education of China (no.6141A02022314).

\section{REFERENCES}

A.Erturk., 2014. Spatial resolution enhancement of hyperspectral images using unmixing and binary particle swarm optimization. IEEE Geoscience \& Remote Sensing Letters 11(12), pp. 21002104.

Atkinson, P. M., 2005. Sub-pixel target mapping from softclassified, remotely sensed imagery. Photogrammetric Engineering \& Remote Sensing 71(7), pp. 839-846.

Boucher, A. and Kyriakidis, P. C., 2006. Super-resolution land cover mapping with indicator geostatistics. Remote Sensing of Environment 104(3), pp. 264-282.

Chang, C. I., 2017. Adaptive linear spectral mixture analysis. IEEE Transactions on Geoscience \& Remote Sensing 55(3), pp. 1240-1253.

Chen, S., Li, X. and Zhao, L., 2017. Subpixel mapping method of hyperspectral images based on modified binary quantum particle swarm optimization. Journal of Electrical and Computer Engineering 2017(3), pp. 1-17.

Fisher, P., 1997. The pixel: A snare and a delusion. International Journal of Remote Sensing 18(3), pp. 679-685.

Ge, Y., Chen, Y., Stein, A., Li, S. and Hu, J., 2016. Enhanced subpixel mapping with spatial distribution patterns of geographical objects. IEEE Transactions on Geoscience \& Remote Sensing 54(4), pp. 2356-2370.
Heinz, D. C. and Chein-I-Chang, 2002. Fully constrained least squares linear spectral mixture analysis method for material quantification in hyperspectral imagery. IEEE Transactions on Geoscience \& Remote Sensing 39(3), pp. 529-545.

Liu, Q. and Trinder, J., 2016. Subpixel mapping of multispectral images using markov random field with graph cut optimization. IEEE Geoscience \& Remote Sensing Letters 13(10), pp. 15071511.

Mertens, K. C., de Baets, B., Verbeke, L. P. C. and de Wulf, R. R., 2006. A subpixel mapping algorithm based on subpixel/pixel spatial attraction models. International Journal of Remote Sensing 27(15), pp. 3293-3310.

Peijun, D. U., Xia, J., Xue, Z., Tan, K., Hongjun, S. U. and Rui, B., 2016. Review of hyperspectral remote sensing image classification. Journal of Remote Sensing 20(2), pp. 236-256.

Shi, W. Z., Zhao, Y. L. and Wang, Q. M., 2014. Sub-pixel mapping based on bp neural network with multiple shifted remote sensing images. Journal of Infrared \& Millimeter Waves 33(5), pp. 527-532.

Thornton, M. W., Atkinson, P. M. and Holland, D. A., 2007. A linearised pixel-swapping method for mapping rural linear land cover features from fine spatial resolution remotely sensed imagery. Computers \& Geosciences 33(10), pp. 1261-1272.

Wang, P., Wang, L. and Chanussot, J., 2016. Soft-then-hard subpixel land cover mapping based on spatial-spectral interpolation. IEEE Geoscience \& Remote Sensing Letters 13(12), pp. 1851 1854.

Xu, X., Zhong, Y. and Zhang, L., 2014. Adaptive subpixel mapping based on a multiagent system for remote-sensing imagery. IEEE Transactions on Geoscience \& Remote Sensing 52(2), pp. 787-804.

Zhao, L., Fan, M., Li, X. and Wang, L., 2015. Fast implementation of linear and nonlinear simplex growing algorithm for hyperspectral endmember extraction. Optik - International Journal for Light and Electron Optics 126(23), pp. 4072-4077. 